\title{
Corela
}

Cognition, représentation, langage

HS-28 | 2019

Les affordances langagières : textualité numérique, matérialité discursive

\section{Affordances et matérialité dans les publications fantômes sur Facebook}

\section{Laetitia Emerit-Bibié}

\section{CpenEdition}

Journals

Édition électronique

URL : http://journals.openedition.org/corela/8486

DOI : $10.4000 /$ corela. 8486

ISSN : 1638-573X

Éditeur

Cercle linguistique du Centre et de l'Ouest - CerLICO

Référence électronique

Laetitia Emerit-Bibié, "Affordances et matérialité dans les publications fantômes sur Facebook », Corela [En ligne], HS-28 | 2019, mis en ligne le 11 septembre 2019, consulté le 13 septembre 2019. URL : http://journals.openedition.org/corela/8486 ; DOI : 10.4000/corela.8486

Ce document a été généré automatiquement le 13 septembre 2019.

\section{cc) (†) (2)}

Corela - cognition, représentation, langage est mis à disposition selon les termes de la licence Creative Commons Attribution - Pas d'Utilisation Commerciale - Partage dans les Mêmes Conditions 4.0 International. 


\title{
Affordances et matérialité dans les publications fantômes sur Facebook
}

\author{
Laetitia Emerit-Bibié
}

1 Les affordances de certaines formes technolangagières (Paveau 2012) interrogent le rapport à la matérialité des énoncés tels qu'ils apparaissent dans les environnements numériques. C'est ce que soulignait déjà Marie-Anne Paveau, en 2010, à propos de la relation objet-discours :

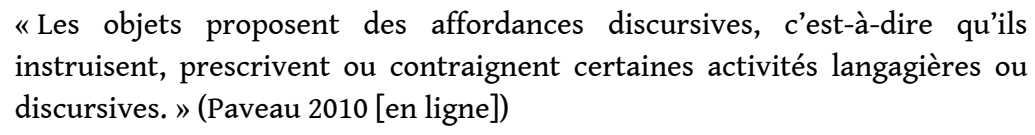

2 Nous tenterons dans cet article de contribuer à creuser cette perspective dans les environnements numériques. Pour cela nous nous appuierons sur la théorie des affordances telle qu'elle a été développée par Gibson (1979), Norman (1988) et Paveau (2010)
«L'affordance est une possibilité offerte par l'objet lui-même, sa forme, sa couleur, sa matière, sa taille, etc., une propriété d'un objet ou un trait de l'environnement immédiat, qui indique quelle relation l'agent humain doit instaurer avec l'objet (comment on doit s'en servir, ce qu'on doit faire avec). » (Paveau 2010 [en ligne])

Dans les environnements numérique cette interaction entre l'environnement et l'utilisateur peut prendre des formes technolangagières, c'est le cas de ce que nous appelons les «publications fantômes » sur Facebook. Nous désignons par «publications fantômes » les énoncés translucides qui n'existent que pour l'utilisateur tant qu'il n'a pas cliqué sur le bouton « publier ».

Dans l'objectif de décrire et de proposer une typologie des publications fantômes sur Facebook nous avons été amené à remonter le chemin descriptif en sens inverse, c'est-àdire de nous poser la question de la matérialité des publications numériques et de leur définition. 
5 En effet, le terme "publication » est à la fois ambigu et polysémique, pourquoi le conserver? Quels sont les traits génériques des publications numériques et sont-ils différents des publications pré-numériques ? Faut-il considérer tout contenu mis en ligne comme une publication numérique? Quels sont les procédés d'éditorialisation et quelles affordances sont communes à toutes les publications? Quel(s) lien(s) peut-on faire entre publication et identité numérique?

6 Nous tenterons de répondre à ces questions au travers d'une approche descriptive d'analyse du discours numérique, pas à pas, parfois de façon laborieuse et d'autres de façon partielle. L'objectif étant de poser les bonnes questions avant d'apporter les réponses et pour ce faire de proposer des outils théoriques nécessaires pour appréhender ces objets complexes.

7 Nous nous appuierons sur une définition et une typologie des publications numériques puis des publications fantômes pour tenter de répondre à ces questions tout en soulevant les interrogations rencontrées au fil de notre travail.

8 Ce travail a été constitué à partir du lieu de corpus « Ma Thèse Sdl » ouvert en 2013 dans le cadre de ma recherche doctorale et d'un micro corpus de captures d'écran recueilli au fil de l'eau sur mes comptes personnels depuis 2015. L'évolution continue des technologies et des environnements numériques ne permet pas une vision exhaustive mais nous tenterons toutefois de donner un aperçu le plus large possible.

\section{De la publication à la publication numérique : enjeux et pluralité lexicales}

9 Le terme de "publication » ou de " publication numérique » est ambigu, polysémique et souvent utilisé pour renvoyer à des réalités multiples qu'elles soient pré ou post numériques. Publier un livre et publier un statut Facebook sont des actions différentes pourtant on peine à trouver dans la littérature une explicitation claire du terme «publication numérique» qui le différencierait réellement de l'acceptation prénumérique du terme " publication».

10 En partant des définitions existantes et de la littérature nous proposerons de décrire les caractéristiques linguistiques et technologiques des publications telles qu'elles apparaissent dans les environnements numériques. Cela nous permettra d'établir une typologie des formes rencontrées dans nos corpus de travail et de nous interroger notamment sur la grille de lecture à appliquer à ces objets technolinguistiques.

\section{Définitions pré-numériques}

11 Nous avons choisi de nous appuyer sur les définitions données par la $9^{e} e$ édition $d u$ dictionnaire de l'Académie Française $e^{1}$ et le Larousse pour l'entrée «publication ». Ces deux dictionnaires de référence nous semblent complémentaires, ils ont tous les deux été mis à jour durant les dix dernières années et sont consultables en ligne ${ }^{2}$.

12 La définition de publication dans la 9e édition du dictionnaire de l'Académie Française mentionne deux sens possibles:

« $خ 1$. Action de rendre une chose publique, notoire, de la porter à la connaissance de tous, en particulier par voie officielle. La publication des 
bans d'un mariage. La publication d'un acte judiciaire dans la presse. Publication des lois et des décrets.

$\succsim 2$. Action de faire paraître, sous forme d'imprimé, une œuvre littéraire ou scientifique, un périodique, etc. ; par méton., l'ouvrage publié. Autorisation de publication. La publication de son ouvrage a été retardée. Le journal intime de cet écrivain est en cours de publication. Établir l'édition critique d'un texte en vue de sa publication. La publication d'un journal, d'une revue. Les publications de la rentrée littéraire. Une publication posthume. Par anal. Publication électronique, publication en ligne. ${ }^{3}$ ”

13 Le Larousse en ligne propose de différencier cinq acceptions du terme " publication »:

1. "Action de porter à la connaissance de tout le monde un acte ou une situation juridique : la publication des bans du mariage. »

2. «Action de rendre publique, de publier une information, une opinion: La publication d'une interview dans un journal. »

3. «Action de publier un texte, une revue, un livre. »

4. «Ouvrage publié et, en particulier, ouvrage périodique, revue.»

5. «Article que publie, en particulier dans une revue, un chercheur, un universitaire, etc. »

On trouve dans les articles en lien avec cette définition «publication assistée par ordinateur" dans les expressions associées au même titre que "directeur de la publication ».

La page consacrée aux synonymes est également intéressante car elle propose les termes " promulgation » (sens 1 et 2), « lancement, parution, sortie » (sens 3 et 5), « ouvrage » (sens 4), ainsi que "édition électronique, microédition» pour le sens PAO qui n'est pourtant pas répertorié dans les définitions mais dans les expressions liées à l'entrée «publication».

On peut noter que le sens 2 est assez proche de celui que l'on attribue généralement à la publication en ligne (et particulièrement sur les réseaux sociaux). Pourtant ce sens précis n'est pas mentionné et on reste sur une définition pré-numérique. On pourrait sans doute parler de définition péri-numérique puisqu'elle coexiste avec l'utilisation du terme dans un contexte numérique bien spécifique. Pourtant cette dernière utilisation du terme dans un contexte numérique natif est comme un point aveugle non pris en compte par les dictionnaires de référence français.

Pour illustrer ce phénomène on peut se pencher sur l'entrée " publication Facebook » sur Google par exemple : 


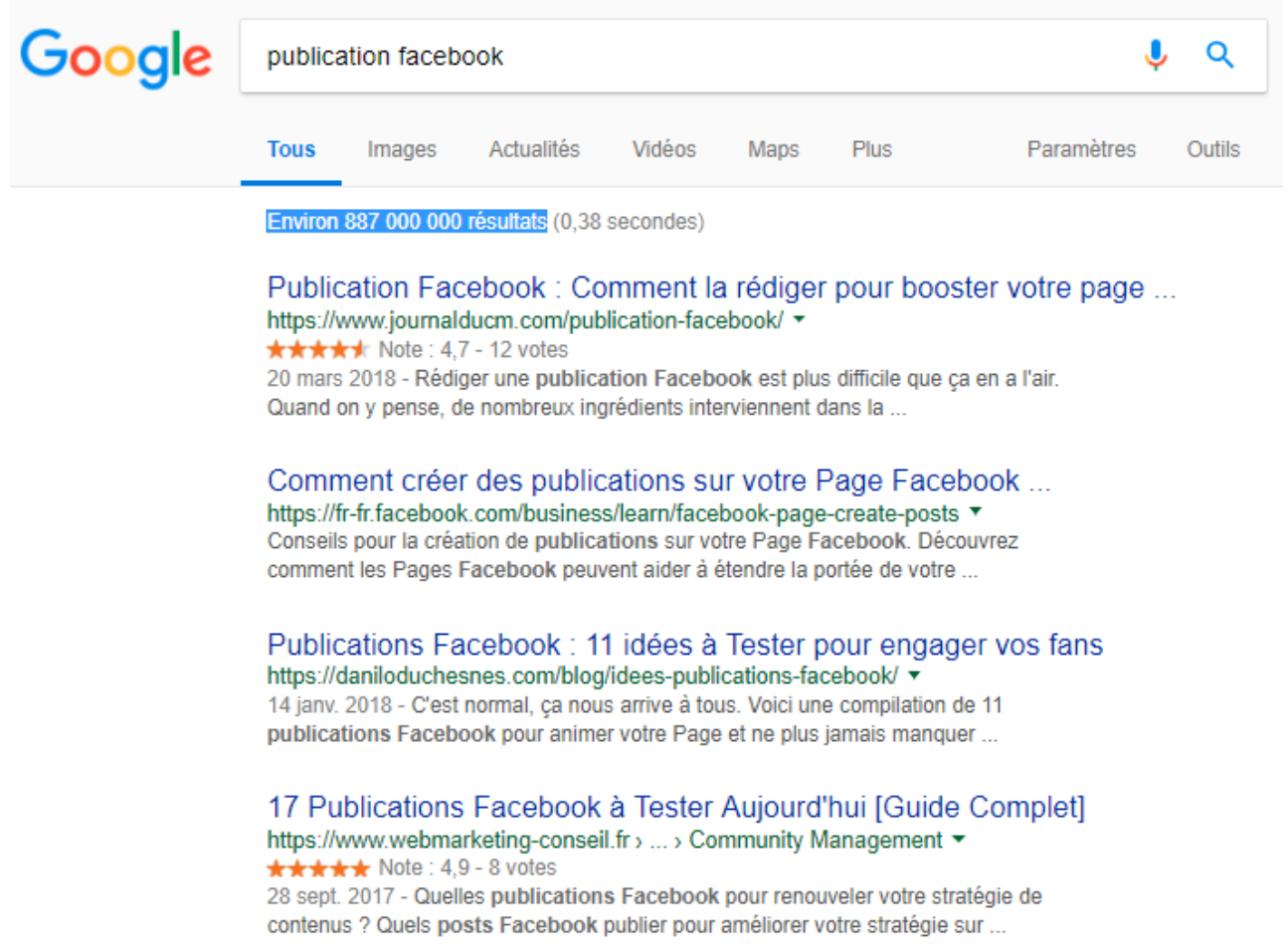

Figure : barre Google capturé le 27/08/18

On le voit dans l'illustration ci-avant, il s'agit d'une expression qui génère environ 887000 000 résultats avec un sens récurrent. Ce sens n'étant pas pris en compte par les deux dictionnaires que nous avons consulté nous nous sommes tournés vers d'autres dictionnaires de langue française et avons notamment retenu celle proposée par l'office québécois de la langue française pour l'entrée « publier » :

« Faire paraître un article dans le réseau Usenet.

Note(s) : Généralement, on publie un article dans Usenet et on envoie un message par courrier électronique.

Le verbe poster n'a pas été retenu, même si, sous l'influence de l'anglais et en contexte de réseau, il est largement utilisé en français dans le sens de "publier ». En effet, le terme poster est un faux ami puisqu'en français, poster n'implique que l'expédition du message et non sa divulgation. ${ }^{4}$ »

19 La référence à l'anglais qui s'explique dans le contexte sociolinguistique québécois fait apparaitre un réel phénomène de concurrence entre les termes « poster/publier » et « post /publication » dans le web francophone. 


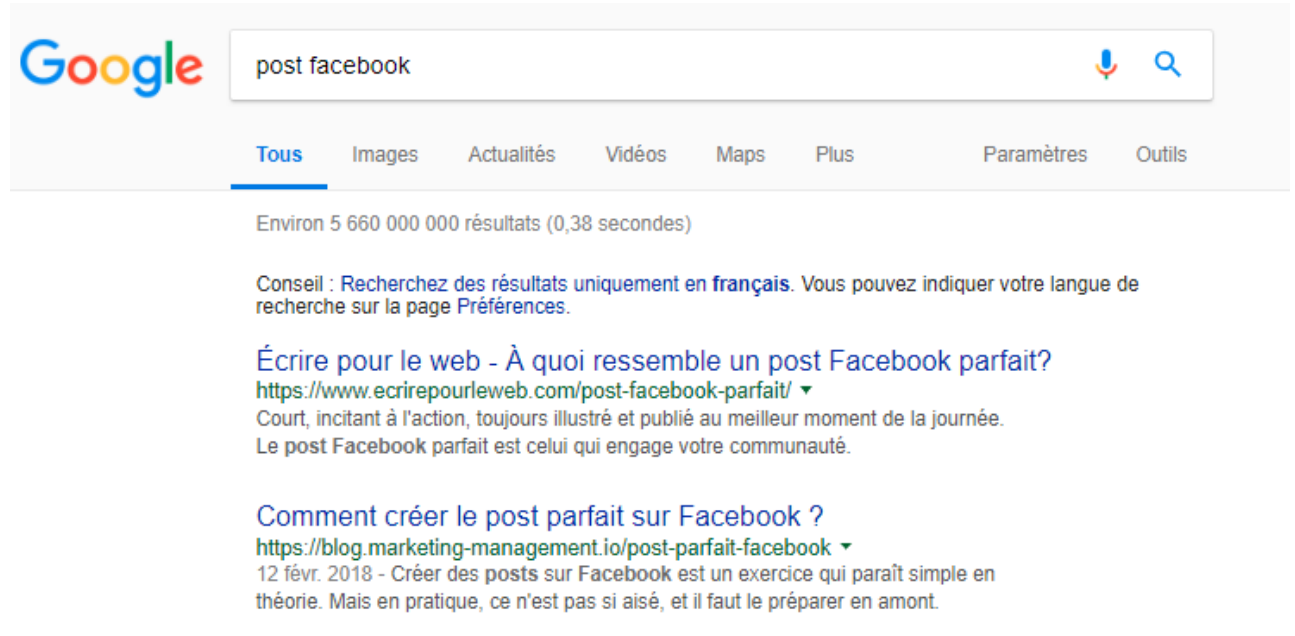

Figure : barre Google capturée le 27/08/18

20 On peut expliquer le nombre de résultats beaucoup plus important pour l'entrée " post Facebook » sur Google par le statut d'emprunt du terme "post » dans de nombreuses langues. On remarque tout de même que, dans les premiers résultats affichés, les emplois de « post » semblent correspondre à ceux de « publication » dans la figure 1.

\section{Vers une définition numérique des publications}

21 Si le terme "publication" est polysémique, c'est également le cas de "publication numérique » dont nous avons trouvé une définition sur Wikipédia uniquement. L'encyclopédie collaborative en ligne propose la définition suivante :

«La publication numérique est l'ensemble des procédés informatiques (micro-ordinateur, logiciels, périphériques d'acquisition, d'écriture...) permettant de produire des documents numériques destinés à être diffusés sur écran : moniteur d'ordinateur, smartphone, tablette, livre électronique, etc. Elle se situe dans la continuité de la PAO, dont l'objectif initial est d'imprimer le document informatique obtenu. ${ }^{5}$ »

22 Ici encore, comme dans l'article du Larousse, on retrouve le lien entre publication et PAO mais aucune mention n'est faite de la potentielle utilisation en traduction directe de l'anglais « post » pourtant attestée en ligne comme on le voit dans les figures 1 et 2.

Pour obtenir cette définition nous proposons de reprendre le deuxième sens donné par le Larousse en ligne «Action de rendre publique, de publier une information, une opinion: La publication d'une interview dans un journal. » en la retravaillant pour y intégrer la dimension numérique. Ce qui donnerait :

"Action de rendre publique, de publier une information, une opinion, notamment au travers de procédés informatiques: La publication d'une interview dans un journal, d'un billet de blog ou d'un statut sur un réseau social numérique.»

24 Cette définition nous donne une base de départ volontairement large à laquelle nous souhaitons ajouter plusieurs caractéristiques proprement numériques qui nous permettent d'appréhender cet objet dans une perspective d'analyse du discours 
numérique. L'analyse du discours numérique, telle qu'elle est proposée par Marie-Anne Paveau, est un cadre théorique en construction dans lequel :

«Le langage est (alors) considéré comme une activité située et non plus autonome, articulée sur les autres activités humaines et inscrite dans l'environnement humain et non humain, et non plus seulement interindividuelle. Dans cette perspective, les objets naturels et artificiels constituent des contributeurs à la production des discours et non plus un simple décor de l'activité de langage. » (Paveau 2012 p. 53)

Dans le dictionnaire des formes et des pratiques de l'analyse du discours numérique (2017), Marie-Anne Paveau s'attache à décrire les commentaires et plusieurs autres formes de publications numériques. Elle propose la définition suivante pour les commentaires :

«Dans une perspective qui considère les énoncés en ligne comme des composites technolangagiers co-intégrant pleinement la dimension technologique et la dimension langagière, le commentaire en ligne peut se définir comme un technodiscours second produit dans un espace dédié scripturalement et énonciativement contraint au sein d'un écosystème numérique connecté. Il est doté d'un certain nombre de traits. " (Paveau 2017, p. 40)

26 Cette définition de "technodiscours second » laisse la place à celle de la publication numérique, telle que nous l'envisageons ici, qui serait un technodiscours premier et dont l'une des caractéristiques serait de pouvoir être augmentée par des commentaires. Le technogenre des publication numériques se compose donc de technodiscours premiers et de technodiscours seconds que nous appellerons ici des publications premières et des publications secondes.

Les technodiscours premiers sont des contenus publiés de façon relativement autonomes ${ }^{6}$ , c'est-à-dire qu'ils existent sans que leur apparition ne soit conditionnée à d'autres publications. Si les technodiscours premiers sont « augmentables » leur apparition n'est pas conditionnée par leur augmentation. Il s'agit par exemple des statuts publiés par les utilisateurs sur Facebook ou des tweets sur Twitter. Ces publications premières affordent des réponses et des commentaires notamment au travers de leurs métadonnées ou de la sémiotique des sites sur lesquels elles apparaissent. Les publications secondes sont dépendantes des publications premières. Un commentaire ne peut apparaître qu'en réaction à une publication première y compris lorsqu'il est délocaliséf

À la suite de la définition des commentaires, Marie-Anne Paveau propose une typologie basée sur leurs traits technolangagiers : « pseudonymat, relationalité, conversationnalité, augmentation et publicité-visibilité ». Elle distingue quatre grandes catégories de commentaires: les commentaires relationnels (qui sont essentiellement d'ordre phatiques), les commentaires conversationnels (qui offrent un contenu discursif), les commentaires délocalisés (qui apparaissent en dehors des espaces dédiés) et les commentaires partagés qu'elle appelle également " pseudo-commentaires ».

Nous reprendrons dans cette analyse les traits technolangagiers décrits par Marie-Anne Paveau au sujet des commentaires puisqu'ils s'appliquent également aux publications premières et aux publications secondes. Par soucis de place et d'efficacité nous renvoyons à la lecture du Dictionnaire des formes et des pratiques et à la description fine et détaillée de chacune de ces caractéristiques que nous relieront simplement ici aux publications premières. 

présence de l'utilisateur et son identité entretiennent une relation paradoxale. L'anonymat n'existe pas en ligne, Louise Merzeau (2010) a montré que la présence numérique est faite de traces volontaires des utilisateurs ou corolaires de l'utilisation de la technologie. On peut donc retrouver dans chaque publication la présence de l'utilisateur qui l'a publiée. Ces traces se situant sur une échelle allant de l'adresse IP à la signature.

De la même manière la relationalité est liée à la nature du web qui est constitué d'un réseau de liens. Chaque publication numérique est liée à d'autres énoncés, à d'autres publications, à d'autres commentaires au sein du même environnement mais également de manière trans-environnementale (Bibié 2017).

32 En termes conversationnels les métadonnées et la matérialité de l'espace dédié se substituent aux marqueurs d'ouverture et de clôture notamment. La présence du bouton « répondre » constitue une affordance conversationnelle qui entraine une récursivité à l'infini.

Le commentaire constitue une augmentation de la publication à laquelle il est rattaché. Il est une augmentation physique puisqu'il prolonge matériellement la publication mais également conversationnelle puisqu'il peut entrainer des réponses voire des modifications de la publication.

trait de publicité-visibilité semble évident, iljustifie le choix du terme "publication» qui évoque l'action de rendre public. Ce trait est particulièrement important car il permet également d'exclure un certain nombre de contenus numériques de la catégorie des publications. Une publication doit posséder, a minima, le trait de publicité-visibilité, même si l'on devrait considérer ce trait comme une graduation variable en fonction des paramétrages des utilisateurs, des fonctionnalités de l'environnement et de l'audience potentielle. Sur Facebook par exemple les utilisateurs peuvent paramétrer leur compte pour décider qui verra leurs publications. Mais il faudrait alors considérer que lorsque l'utilisateur fait le choix de réduire la visibilité d'un contenu à lui-même il ne s'agit pas d'une publication. De la même façon sur Snapchat et Messenger les snaps et les messages qui sont envoyés à des destinataires identifiés ne sont pas des publications, même s'ils sont envoyés à plusieurs destinataires simultanément, puisqu'une messagerie est par essence un environnement privé. Au contraire, les «story» que l'on retrouve par exemple sur Snapchat, Messenger ou encore Instagram, sont "publiées » c'est-à-dire rendues visibles pour les contacts de l'utilisateur.

\section{Typologie non exhaustive des publications numériques}

Pour prendre en compte les publications au-delà des commentaires il nous paraît intéressant d'ajouter trois traits à ceux proposés par Marie-Anne Paveau: l'éditorialisation, l'auctorialité et la collaborativité. Ces trois caractéristiques seront considérées comme des échelles ou des relations, nous parlerons de degré d'éditorialisation et de relation d'auctorialité et de degré de collaboration pour exprimer les nuances existant en pratique dans leur perception par les utilisateurs. Cela nous permettra également d'adapter ces notions pré-numériques sans verser dans une conception binaire. 


\title{
Degré d'éditorialisation
} passent par un processus d'édition et entrent dans la catégorie des publications périnumériques et celles qui n'auraient pas été qualifiées de publication avant les environnements numériques.

Nous désignons ici par publications péri-numériques les publications entrant dans les définitions proposées dans les dictionnaires. Il s'agit de contenus généralement textuels, rendus publics à des fins professionnalisantes (articles, revues, livres numériques, etc.) ou officielles (bans). Nous choisissons de les appeler péri-numériques puisqu'elles peuvent apparaitre indifféremment dans les environnements numériques ou en dehors de ceux-ci bien que l'environnement dans lequel elles sont publiées ait un impact important sur leur contenu. Les publications péri-numériques passent par un processus d'édition avant d'être publiées, c'est une condition même de leur nature de publication.

\begin{abstract}
"L'éditorialisation désigne l'ensemble des dynamiques qui produisent et structurent l'espace numérique. Ces dynamiques sont les interactions des actions individuelles et collectives avec un environnement numérique particulier. » (Vitali-Rosati 2016 [en lingne])
\end{abstract}

Attention cependant, prendre en compte le degré d'éditorialisation ne revient pas à nier l'intervention éditoriale des procédés informatiques de mise en publication et en particulier celle des dispositifs socionumériques et de leurs algorithmes.

\begin{abstract}
«On peut définir l'éditorialisation comme un ensemble d'actions collectives et individuelles, qui ont lieu dans un environnement numérique en ligne, et qui ont pour objectif de structurer notre façon de comprendre, d'organiser et d'interpréter le monde. Ces actions sont façonnées par l'environnement numérique dans lequel elles se réalisent : l'éditorialisation, comme souligné par la première définition, ne prend pas seulement en compte ce que les usagers font, mais aussi comment leurs actions sont déterminées et orientées par un environnement particulier. » (Vitali-Rosati 2016[en lingne])
\end{abstract}

Nous tentons ici de considérer l'éditorialisation comme une graduation qui ne comprendrait pas d'échelon zéro, comme le sous-entend la définition proposée par Marcello Vitali-Rosati. Toutefois il nous semble important de faire une différence entre les contenus ayant subi un processus d'édition (les publications péri-numériques) et dans lesquels les procédés d'éditorialisation sont minorés voire ignorés par une partie des acteurs.

\section{Relation d'auctorialité}

«L'auctorialité est la relation entre l'auteur et sa présence/son implication dans la publication. L'auctorialité est ici étudiée selon trois processus : la production de documents par un ou plusieurs auteurs, la réception par les lecteurs de l'auteur dans l'œuvre ou le document et enfin la liaison permettant de retrouver auteur et document. L'autorité est précisément attachée à l'auctorialité par le lien susceptible d'unir un auteur à un discours ou à un document. » (Broudoux, Bootz, Clément, Grésillaud, Le Crosnier et al., 2005, p. 2) 
40 question de la prise en compte de l'auctorialité dans l'analyse des publications numériques découle du trait d'éditorialisation. Si les publications sont éditorialisées dès lors qu'elles apparaissent dans les environnements numériques, comme nous venons de le postuler à la suite de Marcello Vitali-Rosati, il devient nécessaire de s'interroger sur l'auteur que nous appellerons ici « utilisateur publiant».

Broudoux, Bootz, Clément, Grésillaud, Le Crosnier et al. se sont penchés sur ces questions et proposent de considérer l'action possible des utilisateurs qui reçoivent les publications sur les publications elles-mêmes (éditorialisation) mais également sur l'identité perçue de l'utilisateur publiant :

«Dans le document classique la rencontre du lecteur avec l'auteur est une activité purement mentale que l'on pourrait qualifier de «noématique ». De même que l'auteur lorsqu'il écrit postule un lecteur imaginaire, le lecteur produit par sa lecture une figure imaginaire de l'auteur (qui l'amène bien souvent à confondre l'auteur avec le narrateur). Dans le document numérique, l'interface constitue le lieu privilégié de cette collaboration et l'instrument d'une interaction entre auteur et lecteur. L'activité de lecture n'y est plus purement noématique, elle est aussi manipulatoire. Elle peut être qualifiée d'ergodique dans la mesure où elle suppose un travail sur le dispositif de lecture. L'activité du lecteur n'est plus seulement interprétative, elle participe à la constitution du texte à lire. Cette activité ergodique peut être plus ou moins grande selon les cas, elle est toujours sollicitée par l'auteur dans un dispositif d'interfaçage (icônes, liens hypertextuels, incitations à manipuler le document. " (Broudoux, Bootz, Clément, Grésillaud, Le Crosnier et al., 2005, p. 10)

Les utilisateurs récepteurs des publications deviennent dépositaires d'une partie de l'auctorialité, ils construisent l'identité numérique de l'utilisateur publiant. L'auctorialité est une relation qui entre en jeu dans l'interaction mais aussi dans la perception des publications numériques

\section{Degrés de collaborativité}

Il existe plusieurs possibilités pour créer du contenu en ligne l'utilisateur peut générer des publications de façon individuelle ou collaborative. Nous considérons la notion de collaborativité en dehors de celle d'éditorialisation car il ne s'agit pas d'une affordance des environnements numériques mais bien d'une potentialité exploitée de manière consciente par les utilisateurs.

peut différencier plusieurs degrés de collaborativité le plus léger étant sans doute celui de la collaborativité externe quand un commentaire a une influence sur la publication première (modification de la publication pour donner suite au commentaire). Et le plus fort étant celui que l'on peut observer sur les Wikis dans lesquels les utilisateurs proposent des contenus qui pourront être directement modifiés par les autres.

On voit ici que la temporalité dans le sentiment d'auctorialité est très importante. Elle semble même plus importante que l'interaction. Dans une discussion sur un forum les interactants se sentiront pleinement auteurs de leurs interventions, quand bien même ils pourraient être vus comme une construction $\mathrm{du}$ sens et du texte collaborative (notamment à cause des pratiques de modération), alors que sur Wikipédia le contributeur accepte dès le départ la modification postérieure de son texte par un tiers.

Corela, HS-28 | 2019 
Le degré de collaborativité des publications dépend donc à la fois des affordances environnementales (comment et par qui la publication pourra-t-elle être modifiée) et de la temporalité.

46 Le cas des publications partagées souligne également le rapport des utilisateurs à la collaborativité. La publication première est-elle construite en fonction de partages possibles ? Dans ce cas il faudrait considérer le degré de collaborativité comme constitutif de toutes les publications "partageables». La réponse se trouve sans doute dans les affordances de l'environnement d'apparition de la publication première. Sur Twitter par exemple le «retweet» est une pratique de partage très fréquente et encadrée sémiotiquement et métalinguistiquement par le site. En revanche sur Facebook les paramètres de protection et le fonctionnement concentrique du site peuvent limiter l'utilisation du partage.

\section{Typologie de publications}

47 En prenant en compte les traits technodiscursifs précités il est possible de dresser une première typologie des publications numériques divisées entre les publications périnumériques et les publications numériques. Nous développerons ici la partie concernant les publications numériques et nous la prolongerons dans la suite de l'article en proposant une typologie des publications fantômes.

\section{Publication péri-numériques :}

- Publications hors lignes (livres, revues, bans, etc.)

- Publications numériques éditées (livres numériques, presse en ligne, articles universitaires, etc.)

\section{Publications numériques :}

On peut diviser les publications numériques en deux catégories, les publications premières et les publications secondes. Les publications secondes entretenant un lien de dépendance avec les publications premières comme nous l'avons vu précédemment.

\section{- Publications premières}

*Individuelles (statut FB, tweet, billet de blog) qui présentent une relation d'auctorialité forte autant pour les utilisateurs publiant que pour les utilisateurs recevant la publication. La relation d'auctorialité forte se manifeste également par la forte présence de l'auteur dans ce type de publication.

*Collaboratives (wiki) qui ont un degré élevé de collaborativité y compris en amont de leur publication.

\section{- Publications secondes}

*Dans les publications de type « commentaires » le degré de collaborativité est élevé, les utilisateurs ont conscience de collaborer à quelque chose lorsqu'ils commentent. Ce phénomène est accentué et entretenu par les affordances et les métadonnées des espaces de commentaires qui entraine une grande récursivité (modification de la publication primaire, réponse de l'auteur, etc.).

*Nous différencions les partages (retweets, partages) qui ont un degré de collaborativité assez faible (pas d'intervention dans la publication première et pas de récursivité dans les affordances) contrairement aux commentaires. En revanche l'auctorialité est assez forte 
puisqu'il s'agit de formes cliquables qui permettent d'accéder directement à la publication première.

\section{Le cas des publications fantômes}

\section{Une situation complexe dans la typologie des publication numériques}

Les publications fantômes sont apparues sur Facebook en 2015 avec l'application « souvenirs » notamment. Il s'agit de publications qui apparaissent sur le fil d'actualité de l'utilisateur, elles s'adressent directement au propriétaire du compte et ne sont visibles que de lui tant qu'il ne décide pas de les publier. C'est-à-dire tant qu'il ne clique pas sur "publier ». Les publications fantômes sont donc une forme assez avancée d'affordance technolangagière. Nous choisissons ici de parler de publications «fantômes " pour faire référence au caractère translucide et intangible de ces publication. On pourrait aussi parler de "proto-publications " ou "suggestions de publications " ou encore de publications « prête à publier ».

Nous avons choisi de nous intéresser aux publications fantômes sur Facebook car il s'agit du terrain numérique dans lequel nous les avons le plus observé mais ce type de publications semble exister dans de nombreux environnements. On pourrait le transposer notamment aux billets de blogs avant leur publication ou encore aux commentaires des billets de blogs lorsqu'ils sont proposés à la validation par le propriétaire du blog. Cependant, dans le cas des brouillons de billets de blogs et dans celui des commentaires éditorialisés la question de l'auctorialité n'est pas aussi complexe. On identifie clairement l'auteur dans les deux cas et il reste le même avant et après publication.

61 En regardant la typologie des publications présentée ci-avant on se demande où est ce que l'on pourrait placer les publications fantômes : s'agit-il de publications premières collaboratives dans lesquelles le dispositif socionumérique et l'utilisateur s'allient consciemment pour publier un contenu commun? C'est en tout cas ce que perçoivent les récepteurs. C'est un phénomène qui est assez éloigné du processus d'éditorialisation tel que nous l'avons décrit dans cet article puisqu'il s'agit d'une réelle collaboration, à la manière d'un wiki, au sein de laquelle le dispositif sociotechnique est considéré comme un acteur au même titre que l'utilisateur publiant.

62 Ou bien s'agit-il de publications secondes de type "partage », ce qui est techniquement très proche de ce que perçoit l'utilisateur publiant une publication fantôme? Pourtant contrairement aux partages «classiques » sur Facebook il ne s'agit pas d'une forme cliquable. Selon les formes l'auctorialité du dispositif sociotechnique peut être explicite comme on le voit dans cet exemple : 


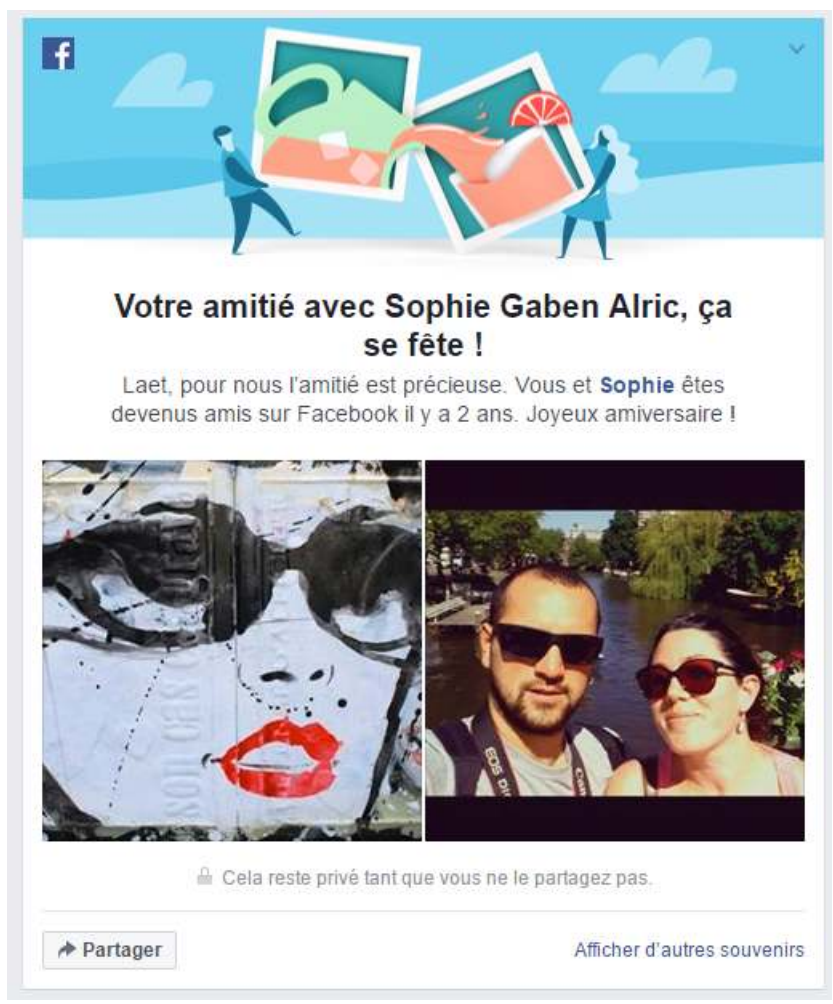

Figure « Amiversaire » capturé le 30/01/2017 compte personnel

63 Il faudrait donc considérer, dans le champ des publications, la publication fantôme comme une publication première collaborative ou une publication secondaire de partage, en fonction du point de vu depuis lequel elle est observée.

\section{Typologie Publications fantômes sur Facebook}

64 Les publications fantômes proposent du contenu plus ou moins élaboré mais dont le point commun est de fournir du «prêt à publier " pour les utilisateurs. Nous avons choisi de présenter les publications fantômes sur Facebook en fonction du type d'occasions qu'elles exploitent. Nous différencierons les évènements particuliers (anniversaires, souvenirs, etc.) qui concernent directement l'utilisateur publiant, et les occasions collectives (fêtes nationales, sport, amiversaires, etc.) qui mettent en lien plusieurs utilisateurs.

\section{Les évènements particuliers}

L'application «souvenirs» (ou «ce jour-là ») développée par Facebook depuis 2015 propose quasiment quotidiennement aux utilisateurs une sélection de publications qui proviennent de son compte ou sur lesquelles il a été identifié. Ce type de publication est assez proche du retweet car elle conserve, une fois publiée, la configuration et les métadonnées d'origine. 


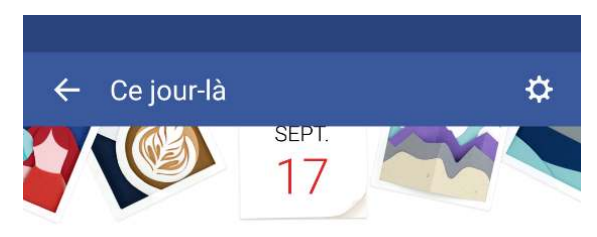

Bonjour Laet ! Nous espérons que vous appréciez ce souvenir d'il y a 3 ans et les autres moments que nous avons retrouvés pour vous aujourd'hui.

\section{f}

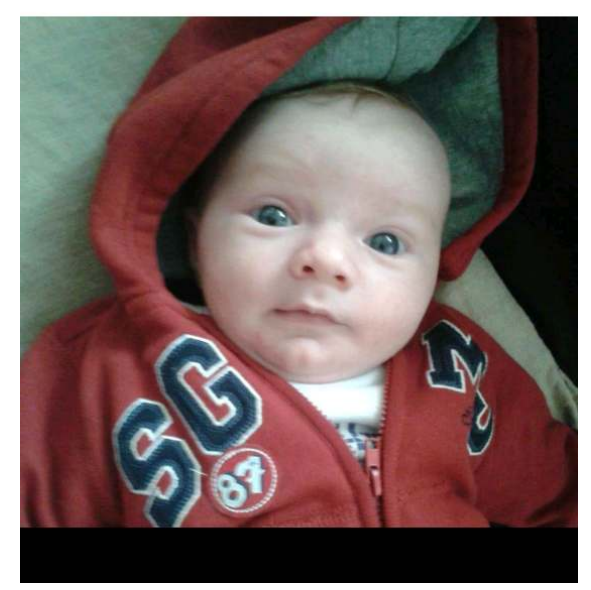

Figure Capturé le 17/09/2016 Smartphone compte personnel

\section{Les occasions collectives}

Cette catégorie est plus vaste et présente des formats plus variés que la première. Nous utilisons le terme « occasions collectives » pour renvoyer aux évènements qui mettent en relation plusieurs utilisateurs sans prendre en compte la nature de ces «occasions ». Il s'agit principalement des fêtes nationales, des deuils, des catastrophes naturelles, des anniversaires de Facebook mais également de ce que le site appelle les « amiversaires ».

67 Voici quelques exemples de formes et de pratiques des publications fantômes autour des occasions collectives telles qu'elles apparaissent sur Facebook. 


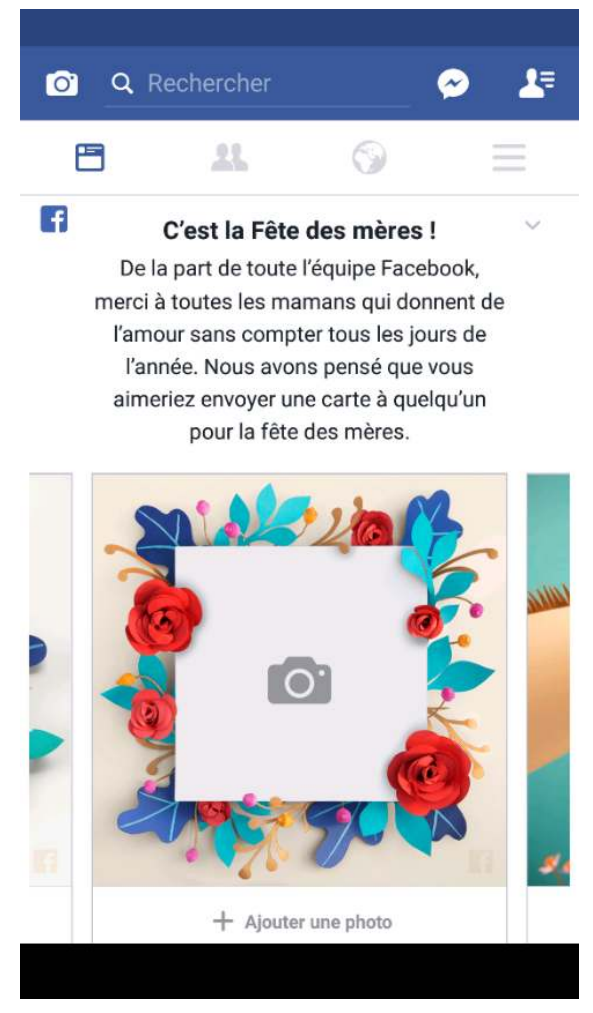

Figure Capturé le 28/05/2017 Smartphone compte personnel

Dans ce premier exemple on voit que la publication fantôme n'est pas ici de nature textuelle, le site propose d'ajouter un filtre à la photo de profil de l'utilisateur pour l'occasion de la fête des mères.

Sur ces trois captures d'écran du 14 juillet 2017 on voit le processus entier de publication d'une publication fantôme :

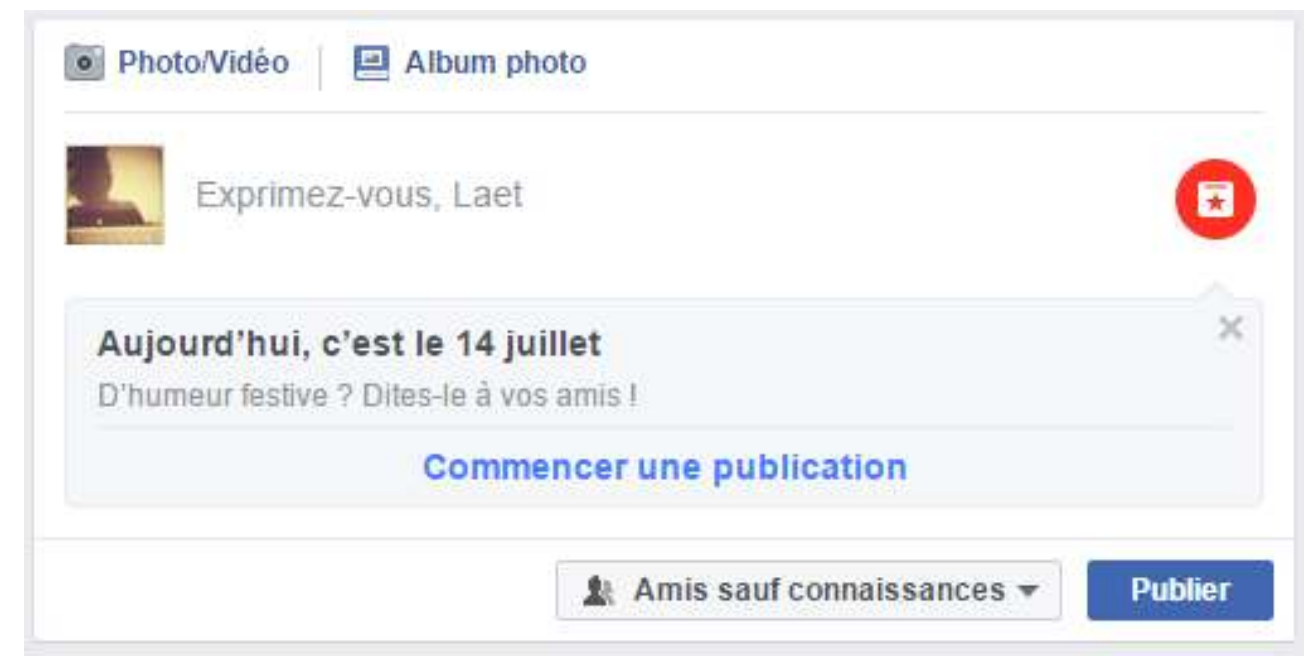

Figure Publication fantôme sans intervention de l'utilisateur

On remarque la sémiotique reconnaissable de l'énoncé cliquable qui apparait en bleu et dont l'affordance technolangagière est presque une prescription. 
-11 fête 14 juillet.

Figure Publication fantôme après intervention de l'utilisateur

71 Une fois que l'utilisateur a cliqué sur le lien il se retrouve dans un espace l'invitant à s'exprimer, encore une fois de façon très prescriptive, et on devine déjà le contenu de la collaboration du site en dessous de la photo de profil.

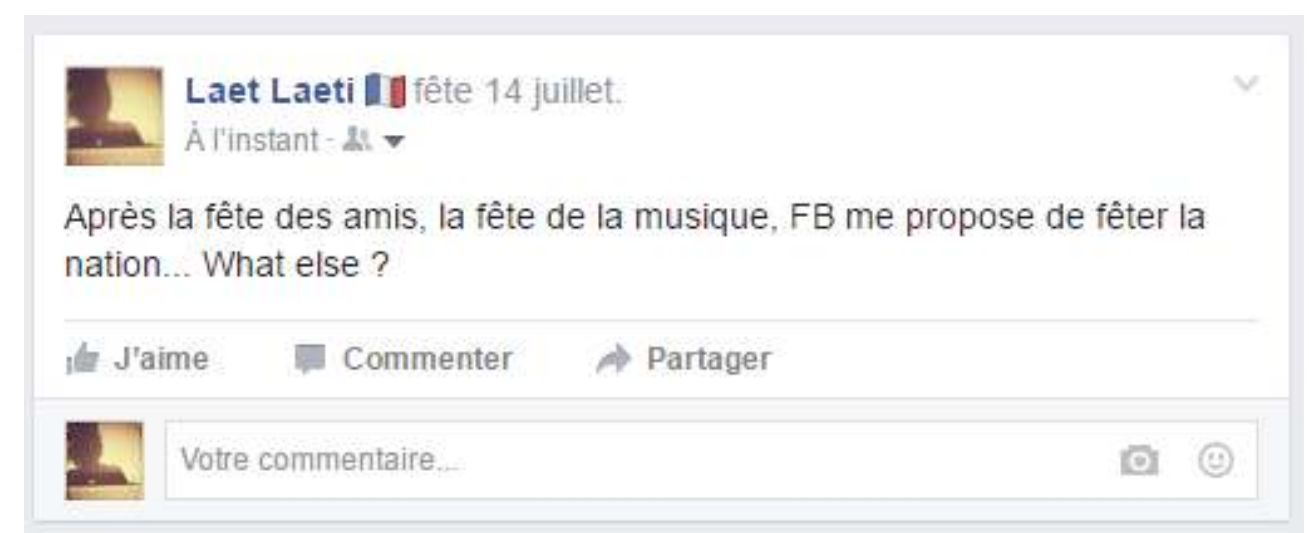

Figure Publication fantôme après publication

72 Une fois publié on retrouve bien le texte provenant du site (drapeau + fête du 14 juillet), puis le texte de l'utilisateur apparait comme n'importe quel autre statut. Dans ce cas, contrairement au souvenir qui s'apparente plus à une publication seconde partagée, on se situe clairement dans une publication première collaborative.

La publication fantôme possède une affordance différente en fonction du point de vue que l'on adopte pour l'appréhender. Est-ce que l'on peut parler d'une idionuméricité de l'affordance de la même manière que l'on parle d'une idionuméricité matérielle des contenus numériques? En tout cas ici l'affordance est clairement située et duelle et a un impact sur la création de contenu et sur la réception de ceux-ci. Le publiant a-t-il conscience que les affordances de réception seront différentes de celles qu'il perçoit quand il publie? Cette dualité est évocatrice des pratiques numériques qui sont focalisées et très situées cognitivement. On pourrait rattacher cette dualité à d'autres pratiques du web et notamment aux questions de violences numériques.

\section{Réflexions conclusives sur le rôle des affordances dans les publications numériques}

La description des publications pose la question de l'auctorialité, on distingue clairement les publications individuelles et les publications collectives tant que l'on se pose la 
question au niveau des utilisateurs mais la place du dispositif sociotechnique est ambiguë et souvent ignorée. Soit parce que la communication des sites à ce sujet est extrêmement opaque (algorithmes secrets) soit par ce que les affordances ne sont pas toujours perçues par les utilisateurs. Interroger l'auctorialité dans les publications numériques soulève la question de l'identité numérique (qui rédige le message ?) et surtout celle de la réception de l'identité numérique ou de la présence numérique (Merzeau, 2010).

L'identité numérique se réparti et s'exprime différemment dans les environnements numériques en fonction des affordances cognitives et sociales qu'ils proposent. C'est l'idée que l'on retrouve dans la notion de double numérique proposée par Jacques Perriault :

« Dès 2003, nous avions proposé la notion de «double numérique » pour caractériser l'ensemble des données que les systèmes d'information recueillent stockent et traitent pour chaque individu, à partir de ses multiples actions via les ordinateurs et les réseaux de télécommunication. » (Perriault, 2009, p. 15)

Il complète cette notion dans la suite de l'article :

«Enfin, les supports utilisés (blogs, sites personnels) comportent des éléments de forme (charte graphique du site, illustrations, etc.) qui contribuent à la définition de soi. La construction de la réputation repose sur l'ensemble des données - contenus et formes - qui structurent la définition de soi et sollicitent le jugement d'autrui. » (Perriault, 2009, p. 17)

Ainsi, l'identité numérique d'un utilisateur est une réalité prise en compte par les autres utilisateurs. Le fait que cette identité numérique corresponde à une identité officielle hors ligne n'a aucune importance. Ce qui compte c'est le degré d'auctorialité et l'implication de l'utilisateur perçu dans la publication. La question de la temporalité est peut-être celle qui montre le plus simplement les choses, si l'on regarde par exemple les comptes/blogs/la présence numérique de personnes décédées (Merzeau 2009) on voit bien que la vie numérique se prolonge bien au-delà de la vie physique. C'est le même phénomène qui permet la présence en ligne de personnages de fiction

L'identité numérique pourrait-elle être considérée comme une affordance du discours numérique lui-même? Il faut pour cela considérer le discours numérique comme métissé de technologie (composite) et admettre le rôle du dispositif à l'intérieur du processus de création du discours (éditorialisation). La nature composite du discours numérique et les différents degrés d'éditorialisation qu'il proposent en font un objet multifaciel à partir duquel les récepteurs déduisent ou construisent l'identité numérique. Ces questions ne trouveront pas de réponses ici mais commencer à les poser semble primordial pour saisir la force des affordances technolangagières dans les environnements numériques. 


\section{BIBLIOGRAPHIE}

Bibié Laetitia, 2017, « La publication multisite sur les réseaux sociaux numériques : Un objet linguistique qui remet en cause la notion de contexte dans les environnements numériques », in Manes Gallo (éd.) Essais, Texte/Contexte : entre autonomie et dépendance : 173-189.

Broudoux E., Bootz P., Clément J., Grésillaud S., Le Crosnier H., et al. 2005, Auctorialité : production, réception et publication de documents numériques. La redocumentarisation du monde.

Gibson, J.J. 1977, « The Theory of Affordances » in R. Shaw and J. Brandsford (éds). Perceiving, Acting, and Knowing: Toward an Ecological Psychology, 62-82.

Gibson, J.J. 1979, The Ecological Approach to Visual Perception, Hillsdale: Lawrence Erlbaum.

Merzeau Louise, 2010, « La présence plutôt que l'identité. Documentaliste », Sciences de

l'Information, ADBS, 47 (1), pp. 32-33.

Norman, D. A, 1988, The design of everyday things, New York, Doubleday.

Paveau Marie-Anne, 2010, "Une linguistique symétrique pour penser le discours », La pensée du discours [carnet de recherche], URL : http://penseedudiscours.hypotheses.org/?p=95

Paveau Marie-Anne, 2012, «Ce que disent les objets. Sens, affordance, cognition », Synergies Pays Riverains de la Baltique $n^{\circ} 9$ : 53-65.

Paveau Marie-Anne, 2017, L'analyse du discours numérique. Dictionnaire des formes et des pratiques, Paris, Editions Hermann, coll. «Cultures numériques ».

Perriault Jacques, 2009, « Traces numériques personnelles, incertitude et lien social », Hermès, La Revue, 2009/1 (n॰53) : 13-20. URL: https://www.cairn.info/revue-hermes-la-revue-2009-1page-13.h

Vitali-Rosati Marcello, 2016, «Qu'est-ce que l'éditorialisation », Sens public [En ligne], URL : http://sens-public.org/article1184.html

\section{NOTES}

1. Il s'agit du tome 3 « de Maquereau à Quotité » qui a été publié en 2011.

2. Dictionnaire de français Larousse [En ligne] consulté le 27/08/2018 à l'adresse suivante: https://www.larousse.fr/dictionnaires/francais/publication/64958

$9 e$ édition du dictionnaire de l'Académie française [En ligne] consulté le 27/08/2018 à l'adresse suivante : http://www.cnrtl.fr/definition/academie9/publication

3. Ibid.

4. Bibliothèque virtuelle de l'office québécois de la langue française [En ligne] consulté le 27/08/2018 à l'adresse suivante: http://www.oqlf.gouv.qc.ca/ressources/bibliotheque/ dictionnaires/Internet/fiches/8352696.html

5. Consulté le 21/08/18 à 15 h51 : https://fr.wikipedia.org/wiki/Publication_num \%C3 \%A9rique

6. Ici le caractère "autonome » désigne la nature de la relation de la publication première aux autres types de publication et non celles qu'elle entretient avec son environnement numérique. 
7. Selon la typologie proposée par Marie-Anne Paveau (2017) certains commentaires sont dits « délocalisés » lorsqu'ils n'apparaissent pas dans les espaces numériques dédiés.

\section{RÉSUMÉS}

Les affordances de certaines formes technolangagières (Paveau 2012) interrogent le rapport à la matérialité des énoncés tels qu'ils apparaissent dans les environnements numériques. C'est le cas de ce que nous appelons les " publications fantômes " sur Facebook. Énoncés translucides qui n'existent que pour l'utilisateur tant qu'il n'a pas cliqué sur le bouton « publier ». Cet article proposera une définition des publications fantômes s'appuyant sur une typologie diachronique réunie à partir d'un micro-corpus constitué depuis leur apparition sur le site en 2015. Cette typologie nous permettra de considérer l'augmentation des affordances et leur utilisation stratégique par Facebook.

Affordances of technolanguage forms (Paveau 2012) question the relation to the materiality of statements as they appear in digital environments. This is the case of what we call "ghost publications" on Facebook. Translucent statements that only exist for the user as long as they have not clicked on the "publish" button. This article will propose a definition of the ghost publications based on a diachronic typology from a micro-corpus constituted since their appearance on the site in 2015. This typology will allow us to consider the increase of the affordances and their strategic use by Facebook.

\section{INDEX}

Mots-clés : Facebook, Analyse du Discours numérique, technologie langagière, réseaux sociaux numériques et affordance

Keywords : Facebook, digital speech analysis, technoligical language, social network, affordances.

\section{AUTEUR}

\section{LAETITIA EMERIT-BIBIÉ}

Université Bordeaux Montaigne 